\title{
Analytical investigation on 3D non-Boussinesq mountain wave drag for wind profiles with vertical variations *
}

\author{
TANG Jin-yun (唐锦涢)，TANG Jie (汤杰)，WANG Yuan (王元) \\ (Department of Atmospheric Science, Key Laboratory of Mesoscale Severe Weather \\ of Ministry of Education, Nanjing University, Nanjing 210093, P. R. China) \\ (Communicated by LU Chuan-jing)
}

\begin{abstract}
A new analytical model was developed to predict the gravity wave drag (GWD) induced by an isolated 3-dimensional mountain, over which a stratified, nonrotating non-Boussinesq sheared flow is impinged. The model is confined to small amplitude motion and assumes the ambient velocity varying slowly with height. The modified Taylor-Goldstein equation with variable coefficients is solved with a Wentzel-KramersBrillouin (WKB) approximation, formally valid at high Richardson numbers. With this WKB solution, generic formulae of second order accuracy, for the GWD and surface pressure perturbation (both for hydrostatic and non-hydrostatic flow) are presented, enabling a rigorous treatment on the effects by vertical variations in wind profiles. In an ideal test to the circular bell-shaped mountain, it was found that when the wind is linearly sheared, that the GWD decreases as the Richardson number decreases. However, the GWD for a forward sheared wind (wind increases with height) decreases always faster than that for the backward sheared wind (wind deceases with height). This difference is evident whenever the model is hydrostatic or not.
\end{abstract}

Key words gravity wave drag (GWD), wind shear, Wentzel-Kramers-Brillouin (WKB) approximation, circular bell-shaped mountain

Chinese Library Classification O175.8, O351.2

2000 Mathematics Subject Classification 34B15, 34B40

Digital Object Identifier(DOI) $\quad 10.1007 / \mathrm{s} 10483-007-0305-\mathrm{Z}$

\section{Introduction}

It is well recognized that the orographic gravity wave drag (GWD) depends critically on the vertical structure of the ambient wind profiles ${ }^{[1]}$. In a recent paper, Teixeira, et al ${ }^{[2]}$ investigated the GWD induced by a 3-dimensional (3D) mountain when a stratified Boussinesq flow crosses it over. Their results show that the vertical variations of the ambient wind influence the GWD greatly. However, we here reinvestigated analytically the behavior of GWD in the presence of shear and curvature in wind profiles for a non-Boussinesq flow. Our results show that some phenomena discovered in numerical simulations but failed to be explained by the Boussinesq model in Ref.[2] become quite clear in the present framework.

The structure of this work is arranged as following: In Section 1, the Taylor-Goldstein equation (i.e, the equation of the vertical velocity perturbation) with variable coefficients is solved to the second order accuracy using a WKB approximation ${ }^{[2]}$. Then in Section 2, analytical formulae are presented for both the GWD and surface pressure perturbations (where the former is balanced by the latter according to Newton's third law on mechanics ${ }^{[1]}$ ) in the presence

\footnotetext{
* Received Oct.18, 2005; Revised Oct.31, 2006

Project supported by the National Basic Research Program of China (973 Program) (No.2004CB418301) and the National Natural Science Foundation of China(Nos.40575017 and 40333031)

Corresponding author WANG Yuan, Professor, Doctor, E-mail: yuanasm@netra.nju.edu.cn
} 
of vertical variations in wind profiles, and following this, their more compacted forms are obtained under hydrostatic approximation. In Section 3, an ideal test is conducted to prove that for the non-Boussinesq flow, the GWD depends critically not only on the strength of the linear shear but also on its direction; however, this dependence would not be revealed even by the non-hydrostatic Boussinesq model. Finally, simple conclusions are drawn in Section 4.

\section{WKB model}

Without loss of generality, we assume that the 3D non-Boussinesq flow considered is steady and non-rotating, and the dynamics involved is adiabatic and inviscid ${ }^{[3-6]}$. Let $\boldsymbol{U} \equiv(U(z), V(z))$, $\bar{\rho}(z)$ be, respectively, the velocity and density of the ambient flow, which are height dependent and vary nevertheless slowly in vertical; and the non-dimensional height $h \equiv N h_{0} / \| \boldsymbol{U}(z=$ $0) \| \ll 1$, where $h_{0}$ is the maximum height of the mountain, $N$ (constant) is the Brunt-Väisälä (or buoyancy) frequency of the ambient flow, operator $\|\cdot\|$ means taking the modul of a certain vector.

After some manipulations onto the governing equations for the small amplitude motions (see Appendix A), the Taylor-Goldstein equation for the non-Boussinesq flow is given as

$$
\hat{w}_{z z}+\left(\frac{N^{2}}{U_{n}^{2}}-2 \frac{\Gamma_{1} U_{n z}}{U_{n}}-\frac{U_{n z z}}{U_{n}}-\delta_{1} K^{2}-\Gamma_{1}^{2}\right) \hat{w}=0
$$

where $\frac{\partial}{\partial z}$ has been designated by subscript $z, \Gamma_{1}$ is the stratification parameter, and $U_{n}=$ $U \cos \sigma+V \sin \sigma$ is the component of the ambient wind when projected into the direction of the horizontal wave-number vector $\boldsymbol{K} \equiv(k, l)=K(\cos \sigma, \sin \sigma)$. Note, hydrostatic approximation can be included or excluded in Eq.(1) simply by taking $\delta_{1}=0$ or $\delta_{1}=1$.

Since it is assumed in prior that $\boldsymbol{U}$ and consequently $U_{n}$, varies slowly with height, Eq.(1) can be solved analytically using the WKB approximation. Thence, introducing some small parameter $\epsilon$, defining the transform $Z=\epsilon z$ and rewriting Eq.(1) yields

$$
\epsilon^{2} \ddot{\hat{w}}+\left(\frac{N^{2}}{U_{n}^{2}}-2 \Gamma_{1} \frac{\dot{U}_{n}}{U_{n}} \epsilon-\frac{\ddot{U}_{n}}{U_{n}} \epsilon^{2}-\delta_{1} K^{2}-\Gamma_{1}^{2}\right) \hat{w}=0
$$

where $\frac{\partial}{\partial Z}$ has been replaced by $(\cdot)$.

Further, take the wavelike solution satisfying the radiation condition at $Z \rightarrow \infty$ (i.e., the solution whose $\operatorname{Re}(m)$ has the same sign with $\left.U_{n}\right)$ as follows:

$$
\hat{w}(Z)=\hat{w}(Z=0) \exp \left[\mathrm{i} \cdot \epsilon^{-1} \int_{0}^{Z} m(\xi) d \xi\right]
$$

where $m(\xi)=\sum_{j=0}^{\infty} \epsilon^{j} m_{j}(\xi)$ is the vertical wave-number, $\mathrm{i}$ is the imaginary unit. Thus together with the lower boundary condition ${ }^{[2]}$

$$
\hat{w}(Z=0)=\hat{w}(z=0)=\mathrm{i} K U_{n 0} \hat{\eta}(k, l)
$$

where $U_{n 0}$ is the $U_{n}$ when evaluated at $z=0$ (or $Z=0$ ), $\hat{\eta}(k, l)$ is the Fourier transform of mountain shape function $\eta(x, y)$, Eqs.(2), (3) and (4) form a complete boundary value problem of a second order ordinary differential equation (ODE).

Then by entering Eq.(3) into Eq.(2), and sorting the results to the second order $O\left(\epsilon^{2}\right)$, one 
finds

$$
\begin{aligned}
m_{0}= & \frac{N}{U_{n}} L^{1 / 2}, \\
\epsilon m_{1}= & \frac{N}{U_{n}}\left(-\frac{\Gamma_{1} U_{n}}{N} \cdot \frac{U_{n z}}{N} \cdot L^{-1 / 2}-\frac{\mathrm{i}}{2} \frac{U_{n z}}{N} L^{-1}\right), \\
\epsilon^{2} m_{2}= & \frac{N}{U_{n}}\left\{\left[-\frac{U_{n} U_{n z z}}{2 N^{2}} \cdot L^{-1 / 2}+\frac{1}{4}\left(\frac{U_{n} U_{n z z}}{N^{2}}-\frac{U_{n z}^{2}}{N^{2}}\right) \cdot L^{-3 / 2}\right.\right. \\
& \left.+\frac{U_{n z}^{2}}{2 N^{2}} \cdot(5 / 4-L) L^{-5 / 2}-\frac{1}{2}\left(\frac{\Gamma_{1} U_{n}}{N}\right)^{2} \cdot\left(\frac{U_{n z}}{N}\right)^{2} \cdot L^{-3 / 2}\right] \\
& \left.-\mathrm{i}\left[\frac{\Gamma_{1} U_{n}}{2 N} \cdot \frac{U_{n} U_{n z z}}{N^{2}} \cdot L^{-1}+\frac{\Gamma_{1} U_{n}}{2 N} \cdot\left(\frac{U_{n z}}{N}\right)^{2} \cdot(2-L) L^{-2}\right]\right\},
\end{aligned}
$$

where $L=1-\left(\Gamma_{1} U_{n} / N\right)^{2}-\delta_{1}\left(K U_{n} / N\right)^{2}$.

Obviously, with Eqs.(5)-(7), it is easy to verify that $\epsilon \backsim\left|U_{n z} / N\right| \backsim R i^{-1 / 2}$, thus the validity of this WKB approximation formally requires large Richardson number of the flow.

\section{Orographic drag}

\subsection{Computation of GWD}

The GWD is computed with the following formula ${ }^{[7]}$ :

$$
\boldsymbol{F}_{\mathrm{d}}=\int_{-\infty}^{\infty} \int_{-\infty}^{\infty} p^{\prime}(z=0) \nabla_{H} \eta d x d y=\rho_{0} \int_{-\infty}^{\infty} \int_{-\infty}^{\infty} p(z=0) \nabla_{H} \eta d x d y,
$$

where in the second formula, transform Eq.(A7) (see Appendix A) is incorporated, and $\nabla_{H} \equiv$ $\left[\frac{\partial}{\partial x}, \frac{\partial}{\partial y}\right]$.

In Fourier space ${ }^{[8]}$, Eq.(8) becomes

$$
\boldsymbol{F}_{\mathrm{d}}=\mathrm{i} \rho_{0} \int_{0}^{\infty} \int_{0}^{2 \pi}(\cos \sigma, \sin \sigma) \hat{p}^{\star}(z=0) \hat{\eta} K^{2} d \sigma d K,
$$

where the asterisk means taking the complex conjugate.

To compute Eq.(9), we then related the reduced pressure perturbation $\hat{p}$ to the vertical velocity perturbation $\hat{w}$ (it is obtained by neglecting the first term in the bracket on the left hand side of Eq.(A8) in Appendix A) as

$$
\hat{p}=\mathrm{i} U_{n}\left[\left(\frac{U_{n z}}{U_{n}}+\Gamma_{1}\right) \hat{w}-\hat{w}_{z}\right] .
$$

Thus when Eq.(3) is entered into Eq.(10), one obtains

$$
\hat{p}(Z)=\frac{U_{n}}{K}\left(\mathrm{i} \Gamma_{1}+m_{0}\right) \hat{w}+\frac{\epsilon}{K}\left(\mathrm{i} \dot{U}_{n}+U_{n} m_{1}\right) \hat{w}+\frac{\epsilon^{2}}{K} U_{n} \cdot m_{2} \hat{w}=\hat{p}_{0}+\epsilon \hat{p}_{1}+\epsilon^{2} \hat{p}_{2} .
$$

Further, evaluating Eq.(11) at $Z=0$ (i.e., $z=0$ ), and incorporating the lower boundary condition by Eq.(4), yields

$$
\begin{aligned}
& \hat{p}_{0}(Z=0)=U_{n 0}^{2}\left[\mathrm{i} m_{0}(Z=0)-\Gamma_{1}\right] \hat{\eta}(k, l), \\
& \epsilon \hat{p}_{1}(Z=0)=\left\{\mathrm{i} U_{n 0}^{2}\left[\epsilon m_{1}(Z=0)\right]-U_{n 0} U_{n 0 z}\right\} \hat{\eta}(k, l), \\
& \epsilon^{2} \hat{p}^{2}(Z=0)=\mathrm{i} U_{n 0}^{2}\left[\epsilon^{2} m_{2}(Z=0)\right] \hat{\eta}(k, l),
\end{aligned}
$$

where (and henceforth) $U_{n 0 z}$ is the derivative of $U_{n}$ against $z$ when evaluated at $z=0$.

Now it is to claim that the GWD can be explicitly computed to the second order accuracy by simply entering Eqs.(12)-(14) one by one into Eq.(9). Note $\boldsymbol{F}=\sum_{j=0}^{2} \epsilon^{j} \boldsymbol{F}_{\mathrm{d} j}$. 


\subsection{Hydrostatic (or low $F r$ ) approximation}

Under hydrostatic approximation, it holds $\left(K U_{n} / N\right)^{2} \backsim(\|\boldsymbol{U}\| / a N)^{2}=F r^{2} \ll 1^{[2,9]}$.

Further, considering $\left(\Gamma_{1} U_{n} / N\right)^{2} \ll 1^{[5]}$, one thus has $L \approx 1$. When the latter is entered into Eqs.(5)-(7), and thence into Eqs.(12)-(14), one finds

$$
\begin{aligned}
& \hat{p}_{0}(Z=0)=\hat{p}_{0 \mathrm{TMV}}-\Gamma_{1} U_{n 0}^{2} \hat{\eta}(k, l), \\
& \epsilon \hat{p}_{1}(Z=0)=\epsilon \hat{p}_{1 \mathrm{TMV}}-\mathrm{i} \Gamma_{1} U_{n 0}^{2} \frac{U_{n 0 z}}{N} \hat{\eta}(k, l), \\
& \epsilon^{2} \hat{p}_{2}(Z=0)=\epsilon^{2} \hat{p}_{2 \mathrm{TMV}}+\frac{\Gamma_{1}}{2} U_{n 0}^{2}\left[\left(\frac{U_{n 0 z}}{N}\right)^{2}+\frac{U_{n 0} U_{n 0 z z}}{N^{2}}-\mathrm{i} \frac{\Gamma_{1} U_{n 0}}{N}\left(\frac{U_{n 0 z}}{N}\right)^{2}\right] \hat{\eta}(k, l),
\end{aligned}
$$

where the subscript 'TMV' is employed to designate solutions for Boussinesq flow ${ }^{[2]}$ (see Appendix B). Obviously, by setting $\Gamma_{1}=0$, Eqs.(15)-(17) are reduced exactly to Eqs.(39)-(41) for Boussinesq flow in Ref.[2].

Thence, by inserting Eqs.(15)-(17) into Eq.(9), the GWD for the hydrostatic non-Boussinesq flow, is finally obtained as

$$
\begin{aligned}
\boldsymbol{F}_{\mathrm{d} 0}=\rho_{0} N \int_{0}^{\infty} \int_{0}^{2 \pi}(\cos \sigma, \sin \sigma) U_{n 0}|\hat{\eta}|^{2} K^{2} d \sigma d K, \\
\epsilon \boldsymbol{F}_{\mathrm{d} 1=}-\rho_{0} N \int_{0}^{\infty} \int_{0}^{2 \pi}(\cos \sigma, \sin \sigma) U_{n 0}|\hat{\eta}|^{2} K^{2}\left(\frac{\Gamma_{1} U_{n 0}}{N} \frac{U_{n 0 z}}{N}\right) d \sigma d K, \\
\epsilon^{2} \boldsymbol{F}_{\mathrm{d} 2}=-\rho_{0} \frac{N}{8} \int_{0}^{\infty} \int_{0}^{2 \pi}(\cos \sigma, \sin \sigma) U_{n 0}|\hat{\eta}|^{2} K^{2}\left[2 \frac{U_{n 0} U_{n 0 z z}}{N^{2}}\right. \\
\left.+\left(\frac{U_{n 0 z}}{N}\right)^{2}+4\left(\frac{\Gamma_{1} U_{n 0}}{N}\right)^{2}\left(\frac{U_{n 0 z}}{N}\right)^{2}\right] d \sigma d K,
\end{aligned}
$$

where by inspection into Eq.(19), it is easy to prove that in component form, there holds $\operatorname{sgn}\left(\epsilon F_{\mathrm{d} 1 x}\right)=-\operatorname{sgn}\left(U_{0 z}\right)$ and $\operatorname{sgn}\left(\epsilon F_{\mathrm{d} 1 y}\right)=-\operatorname{sgn}\left(V_{0 z}\right)$, where $\operatorname{sgn}(x)$ is the conventional sign function. Thus, we are to assert that the GWD for the forward sheared wind (wind increases with height, e.g. $U_{0 z}>0, V_{0 z}=0$ ) is smaller than that for the backward sheared wind (wind decreases with height, $U_{0 z}<0, V_{0 z}=0$ ). Also, it is noticed when the mountain is axisymmetric $^{[2]}$ (so that $|\hat{\eta}|^{2}$ is independent of $\sigma$ ), the relative magnitudes among $\boldsymbol{F}_{\mathrm{d} 0}, \boldsymbol{F}_{\mathrm{d} 1}$ and $\boldsymbol{F}_{\mathrm{d} 2}$ are constant.

\section{$3 \quad$ An ideal test}

The Fourier spectrum of the circular bell-shaped moutain ${ }^{[2]}$ is

$$
\hat{\eta}=h_{0} a^{2} \exp (-a K)
$$

where $a$ is half width of the mountain.

By entering Eq.(21) into Eqs.(18)-(20) and summing up the results, one obtains

$$
\begin{aligned}
F_{\mathrm{d} x}= & F_{\mathrm{d} x \mathrm{TMV}}+\frac{\pi}{4} \rho_{0} N a h_{0}^{2} U_{0}\left[-\frac{1}{4} \frac{\Gamma_{1}}{N^{2}}\left(3 U_{0} U_{0 z}+2 V_{0} V_{0 z}+V_{0}^{2} \frac{U_{0 z}}{U_{0}}\right)\right. \\
& \left.-\frac{1}{16} \frac{\Gamma_{1}^{2}}{N^{4}}\left(6 U_{0} V_{0} U_{0 z} V_{0 z}+5 U_{0}^{2} U_{0 z}^{2}+3 V_{0}^{2} U_{0 z}^{2}+3 V_{0}^{2} V_{0 z}^{2}+2 \frac{V_{0}^{3}}{U_{0}} U_{0 z} V_{0 z}+U_{0}^{2} V_{0 z}^{2}\right)\right],
\end{aligned}
$$




$$
\begin{aligned}
F_{\mathrm{d} y}= & F_{\mathrm{d} y \mathrm{TMV}}+\frac{\pi}{4} \rho_{0} N a h_{0}^{2} V_{0}\left[-\frac{1}{4} \frac{\Gamma_{1}}{N^{2}}\left(3 V_{0} V_{0 z}+2 U_{0} U_{0 z}+U_{0}^{2} \frac{V_{0 z}}{V_{0}}\right)\right. \\
& \left.-\frac{1}{16} \frac{\Gamma_{1}^{2}}{N^{4}}\left(6 U_{0} V_{0} U_{0 z} V_{0 z}+5 V_{0}^{2} V_{0 z}^{2}+3 U_{0}^{2} U_{0 z}^{2}+3 U_{0}^{2} V_{0 z}^{2}+2 \frac{U_{0}^{3}}{V_{0}} U_{0 z} V_{0 z}+V_{0}^{2} U_{0 z}^{2}\right)\right],
\end{aligned}
$$

where $\left(F_{\mathrm{d} x \mathrm{TMV}}, F_{\mathrm{d} y \mathrm{TMV}}\right)$ is for the Boussinesq model ${ }^{[2]}$ (see Appendix B); $U_{0}$ and $V_{0}$ are surface wind components along $x$ and $y$ axes, and their derivatives are designated by subscript $z$.

From Eqs.(22) and (23), we noticed, aside from that $\boldsymbol{F}_{\mathrm{d} 1}$ depends critically on the shear strength and directions (see terms multiplied by $\Gamma_{1}$ ), that terms multiplied by $\Gamma_{1}^{2}$ together with the conclusions presented in Ref.[2] imply that whenever the wind vector $\boldsymbol{U}$ alters its direction smoothly along with height (e.g. $\boldsymbol{U}(z=0)=\left(U_{0}, 0\right)$, so that $V_{0 z} \neq 0$ ), it always holds $F_{\mathrm{d} y}\left(\backsim V_{0 z}\right) \neq 0$, which suggests a lifting force ${ }^{[10]}$ is exerted in perpendicular to the surface wind vector. (Further discussions on this fact are left to another investigation.) This thence generalizes the assertion by the Boussinesq model in Ref.[2] that when wind vector changes both its direction and magnitude in vertical, a lifting force can be induced.

In the forthcoming ideal test, we took $\Gamma_{1}=N /\left(20 U_{0}\right)\left(10^{-5} \mathrm{~m}^{-1}\right.$ or so), and, to avoid shear instability, $R i$ is restricted to $R i \geq 1 / 4^{[11,12]}$.

Case Directional wind shear, wind rotates with height

The wind profile is

$$
U=U_{0}+\alpha z, \quad V=U_{0},
$$

where $\alpha$ and $U_{0}$ are constants. Therefore, when $R i=N^{2} / \alpha^{2}$ and Eq.(24) are introduced into Eqs.(22) and (23), one yields

$$
\begin{aligned}
& F_{\mathrm{d} x}=F_{\mathrm{d} 0 x}\left[1-\frac{3}{32} \frac{1}{R i}-\operatorname{sgn}(\alpha) \frac{1}{\sqrt{R i}}-\frac{1}{800} \frac{1}{R i}\right] \\
& F_{\mathrm{d} y}=F_{\mathrm{d} 0 y}\left[1-\frac{1}{32} \frac{1}{R i}-\operatorname{sgn}(\alpha) \frac{1}{40} \frac{1}{\sqrt{R i}}-\frac{1}{1600} \frac{1}{R i}\right]
\end{aligned}
$$

where $\left(F_{\mathrm{d} 0 x}, F_{\mathrm{d} 0 y}\right)=\boldsymbol{U}_{0} \rho_{0} N h_{0}^{2} a \pi / 4$ is the leading order GWD in absence of vertical variations in wind profiles.

Here we note $F_{\mathrm{d} y} \neq 0$ because of $V_{0}=U_{0} \neq 0$, and as long as $\alpha \neq 0, F_{\mathrm{d} x} \neq F_{\mathrm{d} y}$, so that $\boldsymbol{F}_{\mathrm{d}}$ misaligns with $\boldsymbol{U}_{0}$. Further, in Fig.1, it shows that under the same shear strength, the GWD

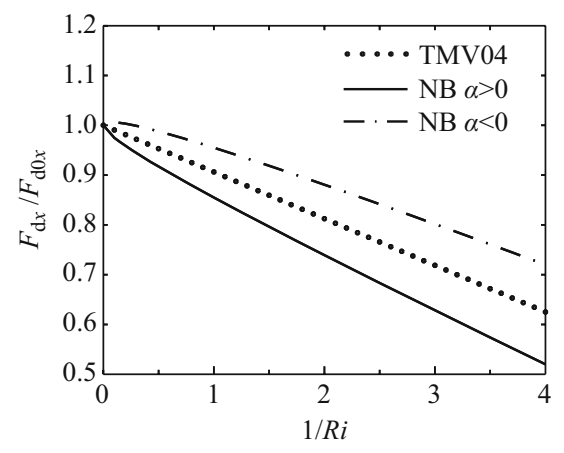

(a) Component in direction $x$

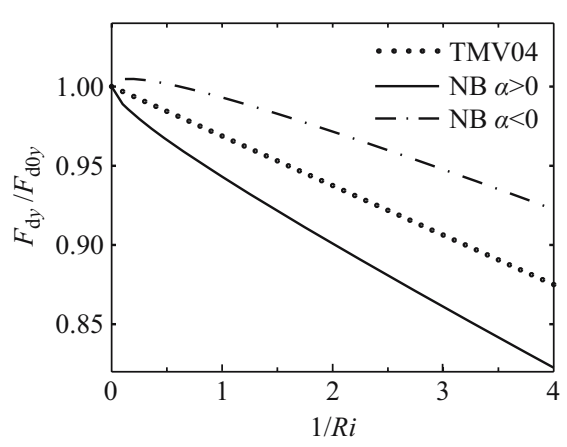

(b) Component in direction $y$

Fig.1 Normalized GWD (computed from Eqs.(25) and (26)) as function of inverse Richardson number $R i^{-1}$ for flow by Eq.(24) (TMV04 denotes Boussinesq model ${ }^{[2]}$, NB denotes the present nonBoussinesq model. The maximum difference between GWD for backward shear $(\alpha<0)$ and that for forward shear $(\alpha>0)$ is about in 0.1 in relative magnitude for both components at $R i=0.25$.) 
for the forward wind shear $(\alpha>0)$ is always smaller than that for the backward wind shear $(\alpha<$ 0 ), and the difference between the two increases as the shear strengthens, being proprotional to $|\alpha| \backsim R i^{-1 / 2}$. While accordingly, in Fig.2(c), we found, just similar with those in Figs.2(a) and $2(\mathrm{e})$, there it shows that the surface pressure perturbation determined by $\operatorname{Im}\left(\hat{p}_{1}\right)$ (i.e the imaginary part of $\hat{p}_{1}$, proportional with $|\alpha|$ ) depicts exactly an asymmetric structure from windward to leeward; and when $\alpha<0$, this asymmetry will add a further pressure gradient force (see Fig.3(e)), pointing from windward to leeward, onto that predicted by Boussinesq model, and when $\alpha>0$ vice versa (see Fig.3(f)). All these rightly explain the puzzle discovered in numerical simulations in Ref.[2], as the shear strengthens, that the GWD for the forward sheared wind decreases much more rapidly than that for the backward sheared wind.

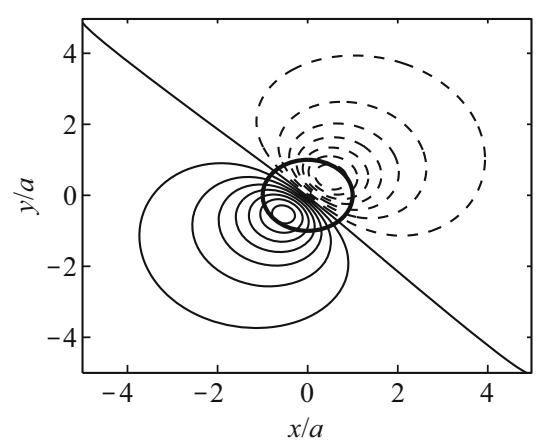

(a) Leading order, $p_{0} /\left(\rho_{0} N h_{0}\left(U_{0}^{2}+V_{0}^{2}\right)^{1 / 2}\right)$

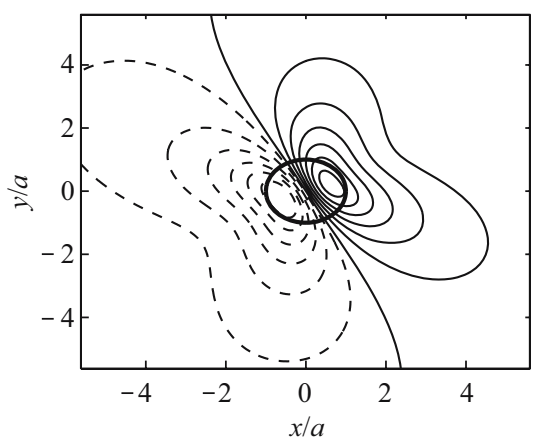

(c) First order, $p_{1 \mathrm{im}} \varepsilon /\left(\rho_{0} N h_{0}\left(U_{0}^{2}+V_{0}^{2}\right)^{1 / 2} R i^{-1 / 2}\right)$

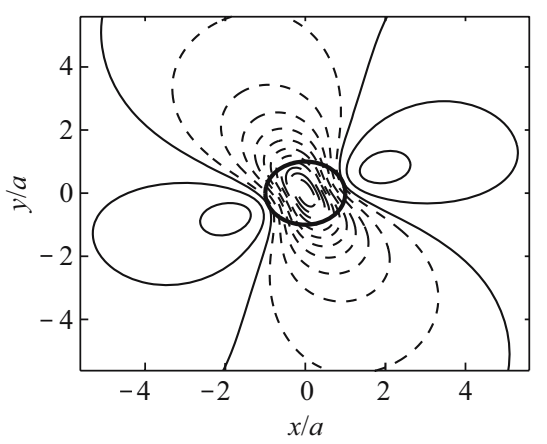

(b) First order, $p_{1 \mathrm{re}} \varepsilon /\left(\rho_{0} N h_{0}\left(U_{0}^{2}+V_{0}^{2}\right)^{1 / 2} R i^{-1 / 2}\right)$

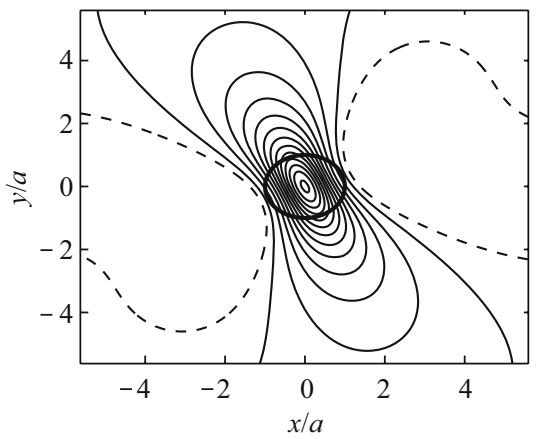

(d) Second order, $p_{2 \mathrm{re}} \varepsilon^{2 /}\left(\rho_{0} N h_{0}\left(U_{0}^{2}+V_{0}^{2}\right)^{1 / 2} R i^{-1}\right)$

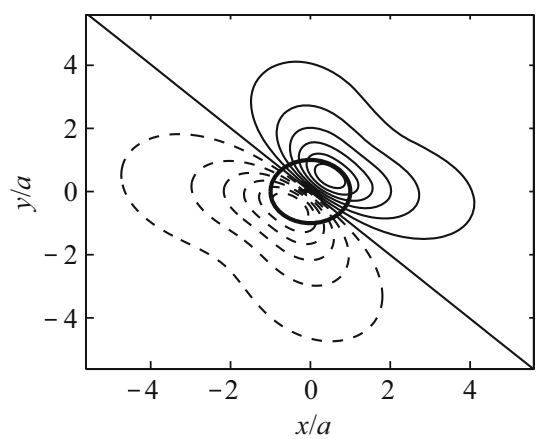

(e) Second order, $\left.p_{2 \mathrm{im}} \varepsilon^{2 /(} \rho_{0} N h_{0}\left(U_{0}^{2}+V_{0}^{2}\right)^{1 / 2} R i^{-1}\right)$

Fig.2 Cross sections of contributions to normalized surface pressure perturbation for flow by Eq.(24) (Thick solid circle denotes mountain evaluation $h_{0} / 2$ at center of domain, solid line denotes positive values; dashed line denotes negative values. Contour spacing from (a) to (e) are 0.05, $0.015,0.0015,0.0005$ and 0.005 . Those subscripted by "re" ("im") are contoured using the real (imaginary) parts of $\hat{p}_{j}, j=1,2$.) 


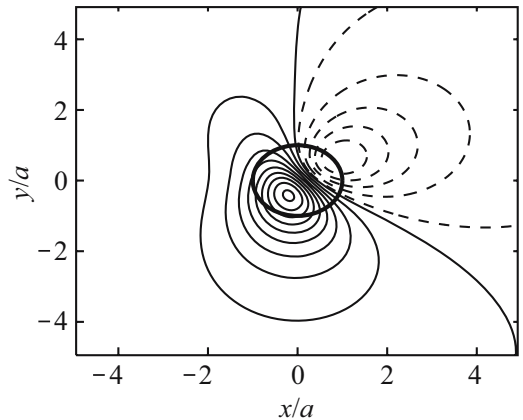

(a) Non-Boussinesq model $(\alpha<0)$, $p /\left(\rho_{0} N h_{0}\left(U_{0}^{2}+V_{0}^{2}\right)^{1 / 2}\right)$

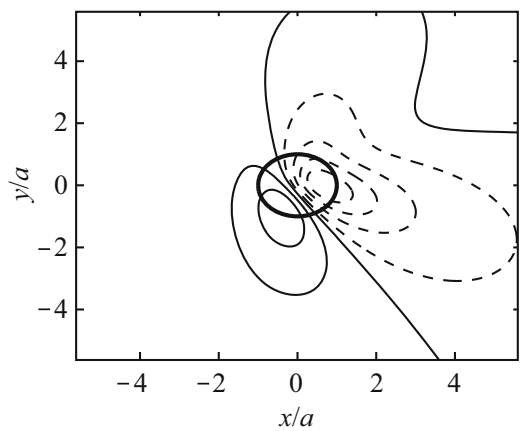

(c) The difference $(\alpha<0)$, $p_{\mathrm{d}} /\left(\rho_{0} N h_{0}\left(U_{0}^{2}+V_{0}^{2}\right)^{1 / 2}\right)$

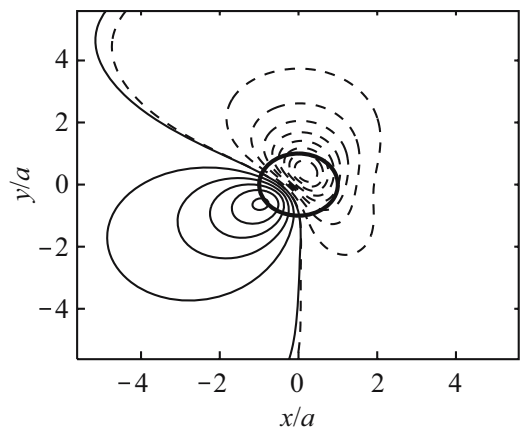

(e) Boussinesq model ${ }^{[2]}(\alpha>0)$, $p_{\mathrm{TMV}} /\left(\rho_{0} N h_{0}\left(U_{0}^{2}+V_{0}^{2}\right)^{1 / 2}\right)$

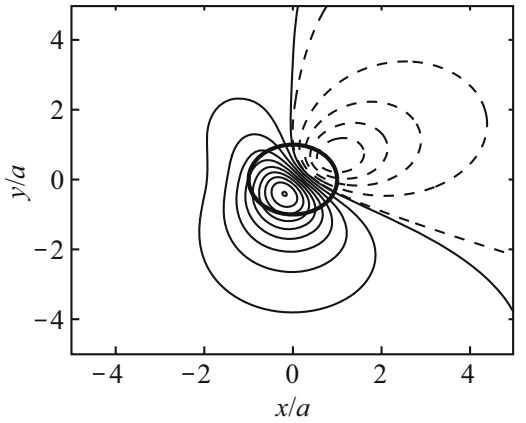

(b) Boussinesq model ${ }^{[2]}(\alpha<0)$, $p_{\mathrm{TMV}} /\left(\rho_{0} N h_{0}\left(U_{0}^{2}+V_{0}^{2}\right)^{1 / 2}\right)$

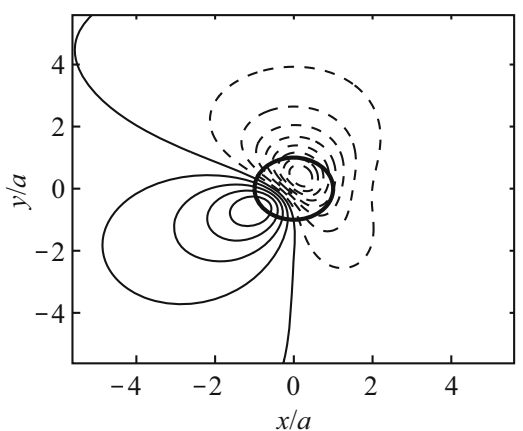

(d) Non-Boussinesq model $(\alpha>0)$, $p /\left(\rho_{0} N h_{0}\left(U_{0}^{2}+V_{0}^{2}\right)^{1 / 2}\right)$

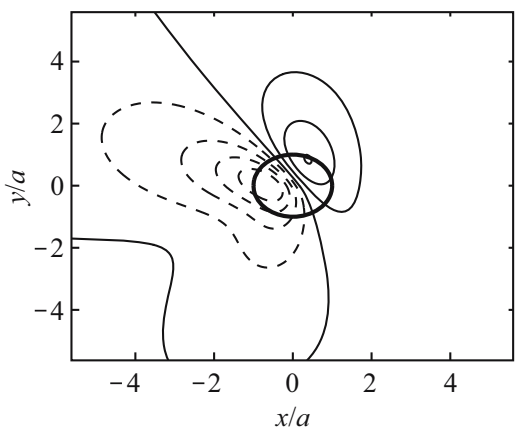

(f) The difference $(\alpha>0)$, $p_{\mathrm{d}} /\left(\rho_{0} N h_{0}\left(U_{0}^{2}+V_{0}^{2}\right)^{1 / 2}\right)$

Fig.3 Cross sections of total contributions to normalized surface pressure perturbation for flow by Eq.(24) when $R i=0.35$ (In Figs.(c) and (f) differences are defined by subtracting results by Boussinesq model from those by present model. Thick solid circle denotes mountain elevation $h_{0} / 2$ at center of domain, solid line denotes positive values, dashed line denotes negative values. Contour spacing from (a) to (c) are 0.05, 0.05, 0.005; from (d) to (e), are 0.05, 0.05 and 0.005.)

\section{Conclusions}

By including the vertical variations of the ambient density (denoted by $\Gamma_{1}$ ), an analytical non-Boussinesq model is developed to predict the orographic GWD. There, both the formulae 
for the GWD and surface pressure perturbation are extended explicitly to the second order accuracy $\left(O\left(R i^{-1}\right)\right)$ in WKB approximation, when the ambient velocity is assumed varying sufficient slowly with height, and by simply setting $\Gamma_{1}=0$, the Boussinesq model ${ }^{[2]}$ can be reproduced exactly.

The results of our theoretical analysis and the ideal test show that the non-Boussinesq model here is more useful than the Boussinesq model ${ }^{[2]}$ in explaining some physical facts discovered in research.

\section{References}

[1] Blumen W. A random model of momentum flux by mountian waves[J]. Geofys Publ, 1965, 26(2):133.

[2] Teixeira M A C, Miranda P M A, Valente M R, et al. An analytical model of mountain wave drag for wind profiles with shear and curvature[J]. J Atmos Sci, 2004, 61(9):1040-1054.

[3] Queney P. The problem of air flow over mountians: a summary of theoretical studies[J]. Bull Amer Meteor Soc, 1948, 29(4):16-26.

[4] Scorer R S. Theory of waves in the lee of mountains[J]. Quart J Roy Meteor Soc, 1949, 75(2):41-56.

[5] Smith R B. The influence of mountains on the atmosphere[J]. Advances in Geophysics, 1979, 21(3):87-230.

[6] Smith R B. Linear theory of stratified hydrostatic flow past an isolated mountain[J]. Tellus, 1980, 32(4):348-364.

[7] Bretherton F P. Momentum transport by gravity waves[J]. Quart J Roy Meteor Soc, 1969, 95(404):213-243.

[8] Brigham E O. The fast fourier transform[M]. Liu Qun (tranl). Shanghai: Shanghai Sci \& Tech Press, 1979, 72-75.

[9] Drazin P G. On the steady flow of a fluid of variable density past an obstacle[J]. Tellus, 1961, 13:239-251.

[10] Landau L D, Lifshitz E M. Fluid mechanics[M]. 2nd Edition, Vol 6. Butterworth-Heinemann: Pergamon Press, 1987, 3-4, 29.

[11] Booker J, Bretherton F P. The critical layer for internal gravity waves in a shear flow $[\mathrm{J}]$. J Fluid Mech, 1967, 27(3):513-539.

[12] Miles J W. On the stability of heterogeneous shear flows[J]. J Fluid Mech, 1961, 10(4):496-509.

[13] Durran D R. Improving the anelastic approximation[J]. J Atmos Sci, 1989, 46(11):1453-1461.

\section{Appendix A Taylor-Goldstein equation}

When the momentum equation, continuity equation and thermal dynamic equation ${ }^{[10]}$ for the steady, adiabatic, inviscid and no-rotating flow are linearized, the governing equations for the perturbations are given as

$$
\begin{aligned}
& U \frac{\partial u^{\prime}}{\partial x}+V \frac{\partial u^{\prime}}{\partial y}+w^{\prime} U_{z}=-\frac{1}{\bar{\rho}} \frac{\partial p^{\prime}}{\partial x} \\
& U \frac{\partial v^{\prime}}{\partial x}+V \frac{\partial v^{\prime}}{\partial y}+w^{\prime} V_{z}=-\frac{1}{\bar{\rho}} \frac{\partial p^{\prime}}{\partial y} \\
& \delta_{1}\left(U \frac{\partial w^{\prime}}{\partial x}+V \frac{\partial w^{\prime}}{\partial y}\right)=-\frac{1}{\bar{\rho}} \frac{\partial p^{\prime}}{\partial z}-\frac{\rho^{\prime}}{\bar{\rho}} g \\
& U \frac{\partial}{\partial x}\left(\frac{\rho^{\prime}}{\bar{\rho}}\right)+V \frac{\partial}{\partial y}\left(\frac{\rho^{\prime}}{\bar{\rho}}\right)+w^{\prime} S+\frac{\partial u^{\prime}}{\partial x}+\frac{\partial v^{\prime}}{\partial y}+\frac{\partial w^{\prime}}{\partial z}=0 \\
& U \frac{\partial}{\partial x}\left(\frac{\theta^{\prime}}{\bar{\theta}}\right)+V \frac{\partial}{\partial y}\left(\frac{\theta^{\prime}}{\bar{\theta}}\right)+\beta w^{\prime}=0 \\
& -\frac{\rho^{\prime}}{\bar{\rho}}=\frac{\theta^{\prime}}{\bar{\theta}}-\frac{1}{c_{s}^{2}} \frac{p^{\prime}}{\bar{\rho}}
\end{aligned}
$$


where $\left(u^{\prime}, v^{\prime}, w^{\prime}\right)$ are the velocity perturbations in $x, y$ and $z$ directions, respectively; $\rho^{\prime}$ is the density perturbation, and $\theta^{\prime}$ is the potential temperature perturbation; others with bar overhead are variables for the ambient flow, depending on $z$ only. In addition, parameter $\Gamma_{1}=-\beta-S / 2$ and $S=\overline{\rho_{z}} / \bar{\rho}$ are defined to designate density stratification (however, throughout the present work, only the former is used), $\beta=\bar{\theta}_{z} / \bar{\theta}=-S-g / c_{s}^{2}=N^{2} / g$, and $c_{s}$ (constant) is the adiabatic acoustic speed.

Then, adopt the following variable transform

$$
(u, v, w, \rho, b)=\sqrt{\frac{\bar{\rho}}{\rho_{0}}}\left(u^{\prime}, v^{\prime}, w^{\prime}, \frac{\rho^{\prime}}{\bar{\rho}}, g \frac{\theta^{\prime}}{\bar{\theta}}\right), \quad p=\sqrt{\frac{\rho_{0}}{\bar{\rho}}} \frac{p^{\prime}}{\rho_{0}},
$$

where $\rho_{0}=\bar{\rho}(z=0)$, some cross eliminations among Eqs.(A1)-(A6) lead to

$$
\begin{aligned}
& \left(\frac{K^{2} U_{n}^{2}}{c_{s}^{2}}-K^{2}\right) \hat{p}=\mathrm{i} K U_{n}\left(\frac{\partial}{\partial z}-\Gamma_{1}\right) \hat{w}-\mathrm{i} K U_{n z} \hat{w}, \\
& \left(-\delta_{1} K^{2} U_{n}^{2}+N^{2}\right) \hat{w}=-\mathrm{i} K U_{n}\left[\left(\frac{\partial}{\partial z}+\Gamma_{1}\right) \hat{p}\right],
\end{aligned}
$$

where the Fourier transform and its inverse ${ }^{[8]}$ have been used, which are

$$
\begin{aligned}
& \hat{\phi}(k, l)=\frac{1}{2 \pi} \int_{-\infty}^{\infty} \int_{-\infty}^{\infty} \phi \exp [-\mathrm{i}(k x+l y)] d x d y \\
& \phi(x, y)=\frac{1}{2 \pi} \int_{-\infty}^{\infty} \int_{-\infty}^{\infty} \hat{\phi} \exp [\mathrm{i}(k x+l y)] d k d l .
\end{aligned}
$$

Here $\mathrm{i}=\sqrt{-1}$ is the imaginary unit and $\hat{\phi}(k, l)$ is the Fourier transform for $\phi(x, y)$.

Thus, by neglecting the first term in the bracket on the left hand side of Eq.(A8) (i.e., filtering off the sound wave ${ }^{[13]}$ ) and then substitution of $\hat{p}$ into Eq.(A9), the Taylor-Goldstein equation of $\hat{w}$ is found as

$$
\hat{w}_{z z}+\left(\frac{N^{2}}{U_{n}^{2}}-2 \Gamma_{1} \frac{U_{n z}}{U_{n}}-\frac{U_{n z z}}{U_{n}}-\delta_{1} K^{2}-\Gamma_{1}^{2}-\Gamma_{1 z}\right) \hat{w}=0 .
$$

Further, take $\Gamma_{1 z}=0^{[5]}$, Eq.(1) in Section 1 is obtained.

Appendix B GWD and surface pressure perturbation for hydrostatic Boussinesq flow The surface pressure perturbation in Fourier spectrum form is

$$
\begin{aligned}
& \hat{p}_{0 \mathrm{TMV}}(Z=0)=\mathrm{i} N U_{n 0} \hat{\eta}(k, l), \\
& \epsilon \hat{p}_{1 \mathrm{TMV}}(Z=0)=-\frac{N U_{n 0}}{2} \frac{U_{n 0 z}}{N} \hat{\eta}(k, l), \\
& \epsilon^{2} \hat{p}_{2 \mathrm{TMV}}(Z=0)=-\frac{\mathrm{i}}{8} N U_{n 0}\left[2 \frac{U_{n 0} U_{n 0 z z}}{N^{2}}+\left(\frac{U_{n 0 z}}{N}\right)^{2}\right] \hat{\eta}(k, l) .
\end{aligned}
$$

The GWD for the circular bell-shaped mountain is

$$
\begin{aligned}
F_{\mathrm{d} x \mathrm{TMV}}= & \frac{\pi}{4} \rho_{0} N a h_{0}^{2} U_{0}\left[1-\frac{1}{32}\left(3 \frac{U_{0 z}^{2}}{N^{2}}+2 \frac{V_{0}}{U_{0}} \frac{U_{0 z} V_{0 z}}{N^{2}}+\frac{V_{0 z}^{2}}{N^{2}}\right)\right. \\
& \left.-\frac{1}{16}\left(3 \frac{U_{0 z z} U_{0}}{N^{2}}+2 \frac{V_{0 z z} V_{0}}{N^{2}}+\frac{V_{0}}{U_{0}} \frac{U_{0 z z} V_{0}}{N^{2}}\right)\right], \\
F_{\mathrm{d} y \mathrm{TMV}}= & \frac{\pi}{4} \rho_{0} N a h_{0}^{2} V_{0}\left[1-\frac{1}{32}\left(3 \frac{V_{0 z}^{2}}{N^{2}}+2 \frac{U_{0}}{V_{0}} \frac{U_{0 z} V_{0 z}}{N^{2}}+\frac{U_{0 z}^{2}}{N^{2}}\right)\right. \\
& \left.-\frac{1}{16}\left(3 \frac{V_{0 z z} V_{0}}{N^{2}}+2 \frac{U_{0 z z} U_{0}}{N^{2}}+\frac{U_{0}}{V_{0}} \frac{V_{0 z z} U_{0}}{N^{2}}\right)\right] .
\end{aligned}
$$

